\title{
Arctic lakes thickness measurement in the Lena River delta based on the Sentinel-1 observations
}

\author{
Yuliya Leskova ${ }^{1}$, Konstantin Muzalevskiy ${ }^{2 *}$, and Zdenek Ruzicka ${ }^{2}$ \\ ${ }^{1}$ Siberian Federal University, , Krasnoyarsk, Russian Federation \\ ${ }^{2}$ Kirensky Institute of Physics of the Siberian Branch of the RAS - Division of Federal Research \\ Center "Krasnoyarsk Scientific Center of the Siberian Branch of the RAS", Krasnoyarsk, Russian \\ Federation
}

\begin{abstract}
In this paper, we investigate the possibilities of ice thickness remote sensing on freshwater lakes based on Sentinel-1 observations. Fr. was selected as a test site. Kurungnakh Island, located in the Lena River delta, was selected as a test site, where 10 lakes with floating and grounded ice were selected. For the test lakes, the time series of backscatting coefficients measured by Sentinel-1 at horizontal-horizontal polarization (EW mode) and at an angle of 18-20 from September 2015 to June 2017 were analyzed. The average lakes ice thickness was estimated based on the Lebedev's model and air temperature according to the European Center for Medium-Range Weather Forecasts (ECMWF ERA5). It is shown that, the dependence between backscattering coefficient and ice thickness (120-140 $\mathrm{cm}$ ) can be described a linear function (with a standard deviation of 13.5$25.3 \mathrm{~cm}$ and a determination coefficient of $0.70-0.79$ ). The preliminary studies shows that the Sentinel-1 radar data is promising for remote sensing of ice thickness on freshwater arctic lakes.
\end{abstract}

\section{Introduction}

Currently, the Sentinel-1 radar data is not used for remote sensing of the ice thickness on Arctic freshwater lakes. At the same time, the the ice lake thickness is a sensitive indicator, reflected the state of the climate system, and an esential climatic variable, recommended by the World Meteorological Organization for global observation [1]. This paper investigates the possibilities of ice thickness remote sensing for a group of freshwater lakes located on the Kurungnakh Island (Lena River Delta) based on Sentinel-1 radar data (operats on a frequency of $5.4 \mathrm{GHz}$, wavelength is about $5.6 \mathrm{~cm}$ ). The backscatter coefficient (RBC) over test lakes was measured by the Sentinel-1 satellite at HH-polarization (horizontalhorizontal) at an angle between 18-20 degrees from September 2015 to June 2017 in EW mode. Radar images were processed in Sentinel Application Platform (SNAP) according to the standard procedure: applying precise orbits, radiometric calibrate, thermal noise removal, speckle filtering ( $5 \times 5$ Lee filter), range Doppler terrain correction.

*E-mail: $\underline{\text { rsdkm@ksc.krasn.ru }}$ 


\section{Modeling lake ice growth}

Due to the fact that the ice thickness $h$ was not measured at the radar observations, the empirical Lebedev's model [2] was used, expressed by the following equation

$$
h=1,33\left(\sum T_{\mathrm{a}}\right)^{0,58}[\mathrm{~cm}],
$$

(1)

here $\sum\left|T_{\mathrm{a}}\right|$ sum of modules of daily negative air temperatures (in degrees Celsius). To calculate $T_{\mathrm{a}}$, the air temperature (on a height of $2 \mathrm{~m}$ ) was used from the European Center for Medium-Range Weather Forecasts (ECMWF ERA5, spatial resolution 0.25 ${ }^{\circ}$ ) [3]. As an example, Fig. 1 shows the calculations of the lake ice thickness in the area of the southern part of the Kurungnakh Island (72.2987 N, 126.1785 E) based on the Lebedev's model with according to ECMWF data for the period 2016-2017. Note that a strong correlation (determination coefficient, $\mathrm{R}^{2}$, is about 0.979 ) and a small error (root-mean square error, RMSE $=2.15^{\circ} \mathrm{C}$ ) between the air temperature calculated on the basis of ECMWF data and measured by the Stolb Island weather station [4], located several kilometers south east of Kurungnah Island (see Fig. 2), which allows to use of ECMWF data to estimate air temperatures in the Arctic regions, where weather station data are not available.

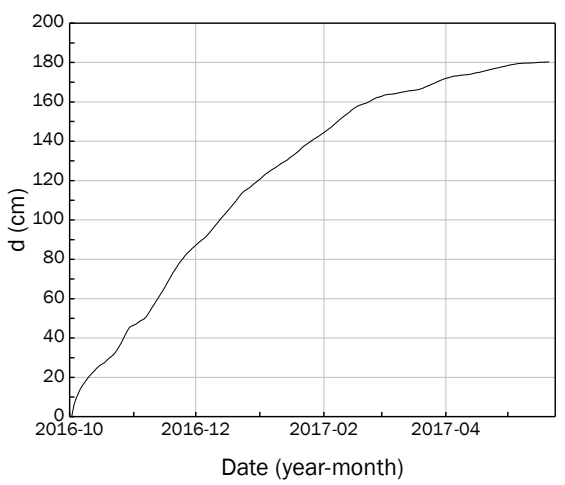

Fig. 1. Ice thickness, calculated based on Lebedev's model, for southern part of Kurungnakh Island (2016-2017).

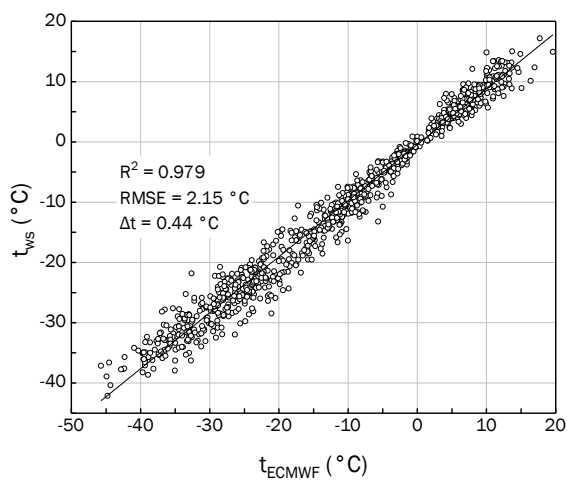

Fig. 2. Daily air temperature between weather station data Stolb Island $\left(T_{\mathrm{ws}}\right)$ and ECMWF ERA5 $\left(T_{\text {ECMwF }}\right)$. 


\section{Method for assessing ice thickness}

The dependence betwen BC measured by Sentinel-1 for a group of shallow lakes (1.05-1.8 $m$ deep [5, see Table 2]) and the ice thickness calculated on the basis of the Lebedev's model (see Fig. 1) is shown in Fig. 3. It can be seen (Fig. 3) that the decrease in BC (occurring due to the contact of the ice to bottom of the lakes) is in good agreement with the depth $d_{\mathrm{i}}$ of the lakes [5, see Table. 2]. From the data shown in Fig. 4, it follows that the ice thickness of the lakes freezing to the bottom, determined on the basis of Sentinel-1 data and Lebedev's model (at the moment when ice touches the lake bottom) with RMSE $=7.4$ $\mathrm{cm}$ and $\mathrm{R}^{2}=0.934$ agrees with ground-based measurements [5, see table. 2]. As a result, it was shown that Lebedev's model can be used to predict the ice lake thickness with an error of no better than $7.4 \mathrm{~cm}$.

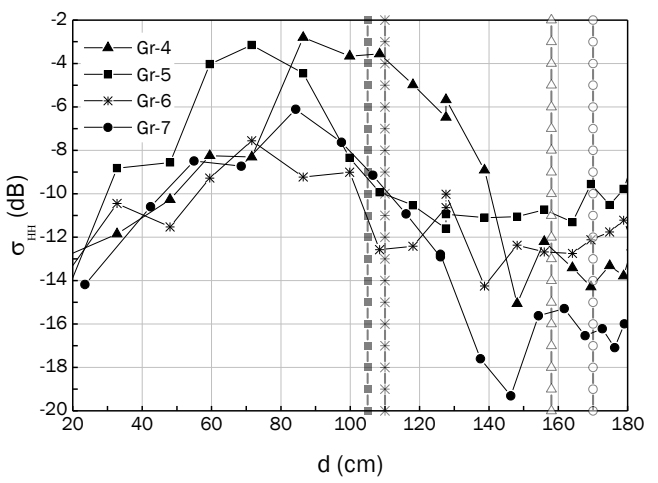

Fig. 3. BC vs ice thickness. The vertical dashed lines mark the depths of the corresponding lakes (highlighted by a symbol) according to the data [5, see table. 2].

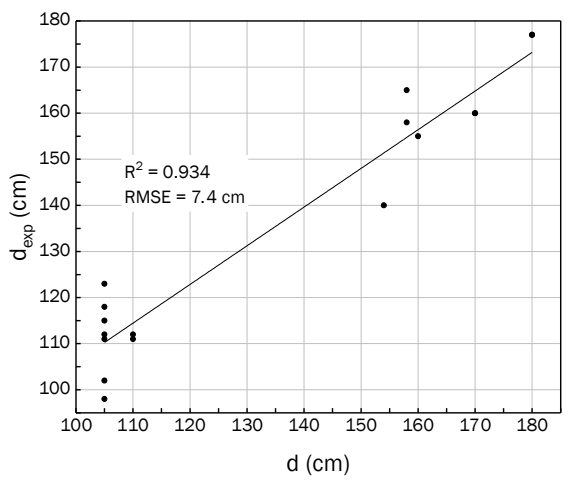

Fig. 4. Dependence between the ice thickness $d_{\exp }$ of lakes freezing to the bottom, estimated according to Sentinel-1 data and Lebedev's model, and the depth of the lakes according to [5, see Table. 2].

In the case of lakes that do not freeze to the bottom, there is a linear relationship between the ice thickness (estimated based on the Lebedev model) and BC, measured by Sentinel-1 at HH polarization (see Fig. 5). During two seasons 2015-2016 and 2016-2017, the RMSE in predicting the lake ice thickness (see [5, Table 2]) based on the BC measured by Sentinel-1 varied from 13.5 to $25.3 \mathrm{~cm}$. The $\mathrm{R}^{2}$ varied from 0.700 to 0.785 (Fig. 5). 

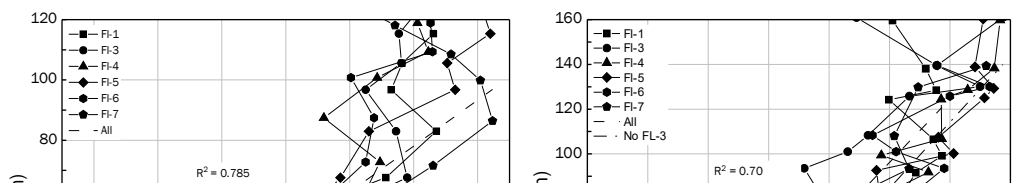

Fig. 5. Dependence between ice thickness calculated on the basis of the Lebedev's model and BC measured by Sentinel-1 at HH polarization in winter: a) 2015-2016 and b) 2016-2017.

\section{Conclusion}

Sentintel-1 data allows to identifying floating and grounded ince in Arctic lakes in the Lena River delta (Kurungnah Island). As a result, on the basis of Sentinel-1 radar data, a remote technology can be proposed for assessing the fish resources of the Arctic lakes, the availability of unfrozen water for industrial needs, and monitoring of climatic changes in the Arctic zone. It is shown that lake ice, up to $120-140 \mathrm{~cm}$ thick, can be determined based on Sentinel-1 data with an error in the range $13.5-25.3 \mathrm{~cm}$.

\section{References}

1. The Global Observing System for Climate: Implementation Needs, GCOS-200, GCOS 2016 Implementation Plan, World Meteorological Organization, 315, Geneva, Switzerland (2016)

2. Lebedev V.V. Ice growth in Antarctic rivers and seas depending on negative air temperatures. Problemy Arktiki -Atctics Problems, 5-6, 9-25, (1938) (In Russian)

3. European Centre for Medium-Range Weather Forecasts. Режим доступа: https://www.ecmwf.int/

4. Stolb weather station data. Available online: https://rp5.lv/Архив_погоды_на_о._Столб

5. S. Antonova, C. R. Duguay, A. Kaab, B. Heim, M. Langer, S. Westermann, J. Boike, Monitoring Bedfast Ice and Ice Phenology in Lakes of the Lens River Delta Using TerraSAR-X Backscatter and Coherence Time Series, Remote Sens, 8(903), 6, (2016) 